\title{
Alterações cognitivas em indivíduos brasileiros com esclerose múltipla surto-remissão
}

\author{
Cognitive profile of Brazilian individuals with \\ relapsing-remitting multiple sclerosis \\ Marco Aurélio Negreiros', Jesus Landeira-Fernandez², Cíntia Villela Kirchmeyer ${ }^{3}$, Renata Alves Paes³ ${ }^{3}$ Regina \\ Alvarenga ${ }^{3}$, Paulo Mattos ${ }^{4}$
}

\section{RESUMO}

Objetivo: O objetivo deste estudo é investigar o perfil neuropsicológico de uma amostra de pacientes brasileiros com esclerose múltipla surto-remissão (EMSR), descrevendo as funções cognitivas mais comprometidas. Métodos: Cinquenta e quatro pacientes com EMSR e 54 controles pareados por sexo, idade e nível educacional foram avaliados por meio de uma longa e abrangente bateria neuropsicológica. Resultados: Observou-se que 59,2\% dos pacientes com EMSR apresentaram algum tipo de comprometimento cognitivo em comparação com o grupo controle. Os testes neuropsicológicos identificaram disfunções executivas,

\section{Palavras-chave \\ Esclerose múltipla, cognição, testes neuropsicológicos.}

\section{Keywords}

Multiple sclerosis, cognition, neuropsychological tests. da memória de longo prazo e da velocidade de processamento de informação. Conclusão: Os resultados encontram-se em conformidade com estudos anteriores relatados na literatura internacional. Contudo, mais estudos ainda são necessários para uma estimativa maior do perfil das alterações cognitivas da esclerose múltipla no Brasil.

\section{ABSTRACT}

Objective: The goal of this study is to investigate neuropsychological profile of a sample of Brazilian patients with relapsing-remitting multiple sclerosis (RRMS) and to describe the cognitive functions more frequently affected. Methods: Fifty-four patients with RRMS and 54 controls matched by sex, age and education were assessed through a long and comprehensive neuropsychological battery. Results: We found that $59.2 \%$ of patients with RRMS had some sort of cognitive impairment as compared to the control group. Neuropsychological testing identified executive functions, long-term memory and speed of information processing as the cognitive functions most frequently impaired among patients. Conclusion: The results are in line with previous studies reported. However, as there are few studies of cognitive impairment in patients with multiple sclerosis in Brazilian literature, more studies are still necessary for a larger evaluation of the cognitive profile of multiple sclerosis in Brazil.

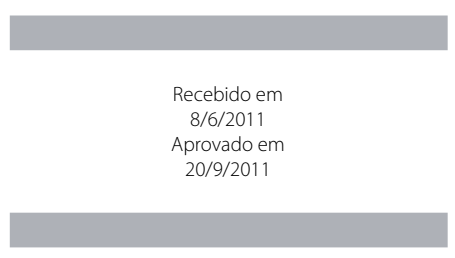

1 Universidade Federal do Rio de Janeiro (UFRJ), Pontifícia Universidade Católica do Rio de Janeiro (PUC-RJ), Departamento de Psicologia, PUC-RJ.

2 PUC-RJ, Departamento de Psicologia; Universidade Estácio de Sá (Unesa), Departamento de Psicologia.

3 Universidade Federal do Estado do Rio de Janeiro (Unirio).

4 UFRJ, Instituto de Psiquiatria.

Endereço para correspondência: Marco Aurélio Negreiros

Rua Rita Ludolf, 17/202, Leblon - 22440-060 - Rio de Janeiro, RJ

E-mail:marconegreiros@superig.com.br 


\section{INTRODUÇÃO}

A esclerose múltipla (EM) é uma doença inflamatória e desmielinizante do sistema nervoso central (SNC), de curso crônico e progressivo, com sintomas de comprometimento disseminado do SNC nas áreas motoras, sensitivas, cerebelares, do tronco cerebral, esfincterianas e mentais ${ }^{1,2}$. Há quatro subtipos clínicos de esclerose múltipla: surto-remissão (EMSR), progressiva primária (PP), progressiva secundária (PS) e progressiva recorrente (PR). Cada um deles possui diferentes perfis clínicos ${ }^{3}$ e fisiopatológicos ${ }^{4}$. O comprometimento cognitivo em cada diferente subtipo parece ser também diferenciado ${ }^{5-7}$.

A disfunção cognitiva na esclerose múltipla caracteriza-se por déficits de memória, atenção, velocidade de processamento de informação e funções executivas ${ }^{8-10}$. O comprometimento cognitivo dos pacientes com EM é considerado moderado quando comparado ao dos indivíduos normais ${ }^{9}$. A prevalência geral de comprometimento cognitivo é de $45 \%$ a $65 \% 8,10,11$.

A avaliação neurológica clínica, assim como outras medidas comuns como a Expanded Disability Status Examination Scale (EDSS) ${ }^{2}$ ou o Mini-Mental Status Examination (MMSE) ${ }^{12}$, é geralmente incapaz de detectar as alterações cognitivas de pacientes com EM ${ }^{8,13}$. Testes neuropsicológicos são os melhores instrumentos para a avaliação da cognição em EM e baterias de testes foram desenvolvidas para esse propósito, 8,14-16.

Diferenças transculturais são consideradas uma importante questão em qualquer pesquisa referente à avaliação da cognição ${ }^{17}$. Há poucos dados disponíveis sobre comprometimento cognitivo na população brasileira de pacientes com EM. Um estudo ${ }^{18}$ foi conduzido com 25 pacientes brasileiros com esclerose múltipla surto-remissão (EMSR), no qual foi constatado que a inteligência geral dos pacientes estava preservada, mas houve um pior desempenho na memória de longo prazo verbal e visual e em tarefas que requisitavam tempo de execução. Paes et al..$^{19}$ investigaram o comprometimento cognitivo em 26 pacientes com EM tipo progressiva primária e encontraram uma frequência de $50 \%$ de comprometimento geral, sendo $60 \%$ de alteração de memória recente, $40 \%$ de alteração de fluência verbal e $40 \%$ de alteração de velocidade de processamento de informação comparativamente ao grupo controle. Em um trabalho prévio ${ }^{20}$, desenvolveu-se uma bateria neuropsicológica breve para identificar as alterações cognitivas em pacientes brasileiros com EMSR composta de três testes cognitivos (Fluência Verbal, Rey Auditorial Verbal Learning Test e Enhanced Cued Recall) com $80,6 \%$ de sensibilidade e 97,2\% de especificidade, encontrando um perfil de alterações cognitivas nesta pesquisa cujo método e resultados serão discutidos a seguir.

No presente artigo, o perfil cognitivo de uma amostra de pacientes brasileiros com EMSR foi investigado por meio de uma abrangente bateria neuropsicológica.

\section{MÉTODOS}

\section{Sujeitos}

Foram selecionados 54 pacientes de um total de 74 consecutivamente admitidos no Setor de Doenças Desmielinizantes do Hospital da Lagoa no Rio de Janeiro. Todos foram diagnosticados com EMSR, clinicamente definida, de acordo com os critérios de Poser et al..$^{21}$ e possuíam níveis leve a moderado de incapacidade - 1 a 6,5 - na Expanded Disability Status Scale (EDSS)2. Quatro critérios de exclusão foram empregados com a finalidade de prevenir possíveis interferências no desempenho nos testes neuropsicológicos: 1) presença de déficit motor ou visual que comprometesse a resposta adequada aos testes cognitivos; 2 ) estado de surto ou piora dos sintomas; 3) uso de substâncias psicoativas, além dos antidepressivos prescritos; e 4) coexistência de condições clínicas que interferissem na cognição. Todos os pacientes passaram por uma entrevista-padrão, análise da ficha médica, exames neurológicos, imagem de ressonância magnética (RM) e exame de líquido cefalorraquidiano, com a finalidade de preencher os critérios mencionados. Seis não preencheram os critérios diagnósticos, cinco tinham EDSS mais alto do que 6,5, três estavam usando substâncias psicoativas, um apresentou surto agudo e cinco não completaram o processo de avaliação.

Cinquenta e quatro adultos saudáveis, pareados de acordo com sexo, idade e escolaridade, formaram o grupo controle. A mesma entrevista aplicada aos pacientes foi conduzida com a finalidade de eliminar participantes que preenchessem algum dos critérios de exclusão. Todos os participantes assinaram o termo de consentimento livre e esclarecido, concordando em participar voluntariamente da pesquisa. Este estudo foi aprovado pelo comitê de ética da Universidade Federal do Rio de Janeiro (UFRJ) e do Hospital da Lagoa em conformidade com os princípios da Declaração de Helsinki.

\section{Testes neuropsicológicos}

Rastreamento de demência: Foi aplicado o teste Memória-Informação-Concentração (MIC), que é útil para esse propósito na EM por não conter tarefas visuais e motoras ${ }^{22}$.

Atenção e velocidade de processamento de informação: Avaliadas pelo Digit Symbol versão oral adaptada por nós para $\mathrm{EM}^{23}$.

Memória de curto-prazo: Avaliada pelo Digit Span Forward e Backward, respectivamente (Bateria WAIS III) ${ }^{24}$.

Memória de longo prazo: Rey Auditory Verbal Learning Test $(\mathrm{RAVLT})^{25}$ foi empregado para medir a memória de longo prazo verbal. Uma fase de recordação livre tardia e outra relacionada ao reconhecimento foram incluídas para que esses dois processos de memória pudessem ser avaliados.

Buschke Enhanced Cued Recall Test (BECR) ${ }^{26}$. Avalia simultaneamente memória verbal e não verbal e a memória de longo prazo com a ajuda de pistas semânticas durante o período de evocação. 
Fluência verbal: Foi testada pelo Controlled Verbal Oral Association Test (COWAT), que mede também a função executiva nos subtestes de produção de palavras fonéticas (F-A-S) e semânticas (animais e frutas) ${ }^{27}$.

Pensamento abstrato: Avaliado por meio da aplicação das séries A e C do teste Raven Progressive Matrices ${ }^{28}$.

\section{Análise dos dados}

As médias dos resultados e seus respectivos desvios-padrão foram apresentados. Um teste T bicaudado foi empregado para detectar diferenças significativas nas médias entre EMSR e grupo controle entre os 26 índices de variáveis cognitivas. $O$ teste do $\chi^{2}$ foi usado para identificar a porcentagem de diferença entre os dois grupos. Uma análise de correlação de Pearson foi usada para acessar a relação entre déficit cognitivo entre os pacientes com EM e a severidade da doença de acordo com o EDSS. Um valor $p$ de 0,05 foi considerado estatisticamente significativo.

Disfunção cognitiva foi definida de acordo com o procedimento empregado por Rao et al. ${ }^{8}$, o qual controla as eventuais diferenças individuais nas habilidades cognitivas pré-mórbidas. A regressão múltipla foi realizada com o escore bruto de cada um dos 26 índices cognitivos como a variável dependente e as variáveis demográficas (sexo, idade e nível educacional) como as variáveis independentes. Como sexo não é uma variável contínua, foi retornado como uma variável falsa. Um padrão residual foi obtido subtraindo da pontuação regressa e da atual para cada um dos 26 índices de variáveis cognitivas. Falha em cada uma dessas variáveis foi definida como o quinto percentil ou abaixo do escore padronizado residual entre o grupo controle pareado.

Déficit cognitivo entre os pacientes com EMSR foi definido como o quinto percentil do número total de falhas nos índices cognitivos dos sujeitos controles. A taxa de frequência de disfunção cognitiva nessa amostra de pacientes EMSR foi calculada como a diferença entre os percentuais de pacientes com EMSR que apresentaram déficit cognitivo a partir de uma taxa de falsos-positivos, definido como a porcentagem de indivíduos controles pareada, erroneamente classificada como cognitivamente alterada.

\section{RESULTADOS}

\section{Dados sociodemográficos}

Não houve diferenças entre idade, escolaridade e sexo entre os dois grupos (todos ps $>0,1$ ). Nossos resultados indicam que a incidência de EMSR nesta amostra foi maior em muIheres $(61,1 \%)$ do que em homens $\left(38,9 \% ; \chi^{2}=7,1, p<0,05\right)$. Pacientes com EMSR apresentaram um maior número de índices fracassados em comparação com os sujeitos controles pareados (t $104=6,67 ; p<0,001$ ).

\section{Frequência das alterações cognitivas}

Empregando um corte de quatro ou mais índices cognitivos fracassados, uma incidência de $59,2 \%$ de pacientes com EMSR e 7,4\% de sujeitos controles foi encontrada com comprometimento cognitivo. Por isso, uma taxa de frequência de comprometimento cognitivo nesta amostra foi estimada em 51,8\%. Curiosamente, nenhuma correlação entre o déficit cognitivo dos pacientes EMSR e a gravidade da doença (EDSS) foi encontrada $(r=0,03, p>0,8)$.

\section{Funções cognitivas e testes alterados}

Os resultados indicaram que os pacientes com EMSR apresentam consistentemente piores desempenhos que os sujeitos controles (todos ps $<0,05$ ) em todos os 26 índices dos testes neuropsicológicos. A tabela 1 apresenta as médias e os desvios-padrão dos 26 índices de variáveis cognitivas.

Fluência verbal fonêmica e semântica foram as variáveis que apresentaram o maior comprometimento. Aproximadamente 40\% dos pacientes com EMSR apresentaram um déficit na geração de palavras que começam com a letra $S$ (fonêmica) ou nomes de frutas (semântica).

A memória de longo prazo, medida pelo RAVLT, também demonstrou um alto índice de comprometimento. Como pode ser observado na tabela 1,35,2\% dos pacientes apresentaram um fracasso na segunda evocação do RAVLT e no teste de reconhecimento, enquanto $33,3 \%$ dos pacientes fracassaram na primeira evocação do RAVLT. Uma considerável sobreposição entre a primeira e a segunda evocação no RAVLT no desempenho entre os pacientes EMRR foi detectada. Somente $17 \%$ dos pacientes que fracassaram no desempenho da primeira evocação foram bem na segunda evocação.

A avaliação da memória de longo prazo que inclui o BECR também demonstrou considerável déficit cognitivo entre os pacientes com EMSR. Aproximadamente 30\% dos pacientes apresentaram um fracasso na primeira tentativa de evocação livre do BECR (BECR-Free 1) e durante toda a evocação (48 pontos) do BECR (BECR-Recall). Esses dois índices não foram redundantes, pois aproximadamente $58 \%$ dos pacientes com EMSR que apresentaram um déficit na primeira tentativa de evocação livre tiveram um bom desempenho durante a evocação do BECR.

\section{DISCUSSÃO}

Os resultados obtidos indicaram que $59,2 \%$ dos pacientes com EMSR apresentaram algum tipo de comprometimento cognitivo. Esse resultado encontra-se em conformidade com estudos anteriores relatados na literatura, os quais indicavam que aproximadamente $45 \%-65 \%$ dos pacientes com esclerose múltipla possuem déficits cognitivos detectados por meio de testes neuropsicológicos $8,10,11$.

Os déficits no pensamento abstrato e na fluência verbal, respectivamente detectados em $27,8 \%$ e $40,7 \%$ dos pacientes, estão relacionados ao comprometimento no funciona- 
Tabela 1. Resultados de testes neuropsicológicos*

\begin{tabular}{|c|c|c|c|c|c|}
\hline Teste cognitivo & EMRS & Controle & $P$ & $\%$ EMRS $<5 \%$ ile & Ordem \\
\hline \multicolumn{6}{|c|}{ Screening de demência } \\
\hline MIC & $2.44(0.40)$ & $1.23(0.26)$ & $<0.014$ & $9.3 \%$ & $21^{\circ}$ \\
\hline \multicolumn{6}{|c|}{ Atenção e concentração } \\
\hline Digit Symbol & $44.37(1.04)$ & $52.81(1.23)$ & $<0.001$ & $22.2 \%$ & $12^{\circ}$ \\
\hline \multicolumn{6}{|l|}{ Memória de } \\
\hline \multicolumn{6}{|l|}{ curto prazo } \\
\hline \multicolumn{6}{|l|}{ Digit Span } \\
\hline Forwards & $5.92(0.15)$ & $6.57(0.18)$ & $<0.008$ & $11.1 \%$ & $20^{\circ}$ \\
\hline Backwards & $3.87(0.15)$ & $4.48(0.21)$ & $<0.018$ & $7.4 \%$ & $22^{\circ}$ \\
\hline \multicolumn{6}{|l|}{ Longo prazo } \\
\hline \multicolumn{6}{|l|}{ RAVL } \\
\hline RAVL 1 & $5.10(020)$ & $6.24(0.23)$ & $<0.001$ & $27.8 \%$ & $8^{\circ}$ \\
\hline RAVL 2 & $7.72(0.33)$ & $8.67(0.34)$ & $<0.05$ & $3.7 \%$ & $23^{\circ}$ \\
\hline RAVL 3 & $9,07(0,35)$ & $10.48(0.34)$ & $<0.005$ & $22.2 \%$ & $12^{\circ}$ \\
\hline RAVL 4 & $10.10(0.37)$ & $11.46(0.31)$ & $<0.007$ & $18.5 \%$ & $18^{\circ}$ \\
\hline RAVL 5 & $10.64(0.34)$ & $12.39(0.30)$ & $<0.001$ & $22.2 \%$ & $12^{\circ}$ \\
\hline RAVL-Recall 1 & $7.56(0.45)$ & $10.39(034)$ & $<0.001$ & $33.3 \%$ & $5^{\circ}$ \\
\hline RAVL-Recall 2 & $8,02(0.44)$ & $10.50(0.38)$ & $<0.001$ & $35.2 \%$ & $3^{\circ}$ \\
\hline RAVL-Recog & $27.29(0.30)$ & $28.63(0.21)$ & $<0.001$ & $35.2 \%$ & $3^{\circ}$ \\
\hline \multicolumn{6}{|l|}{ Buschke } \\
\hline Free 1 & $10.22(0.30)$ & $12.34(0.26)$ & $<0.001$ & $29.6 \%$ & $6^{\circ}$ \\
\hline Free 2 & $11.63(2.12)$ & $13.18(0.26)$ & $<0.001$ & $0.0 \%$ & $24^{\circ}$ \\
\hline Free 3 & $12.11(0.30)$ & $14.16(0.21)$ & $<0.001$ & $20.4 \%$ & $17^{\circ}$ \\
\hline Cued 1 & $5.44(0.25)$ & $3.66(0.26)$ & $<0.001$ & $0.0 \%$ & $24^{\circ}$ \\
\hline Cued 2 & $4.15(0.26)$ & $2.78(0.26)$ & $<0.001$ & $27.8 \%$ & $8^{\circ}$ \\
\hline Cued 3 & $3,72(0.27)$ & $1.74(0.21)$ & $<0.001$ & $0.0 \%$ & $24^{\circ}$ \\
\hline BECR-Recall & $47.27(0.21)$ & $47.94(0.04)$ & $<0.002$ & $29.6 \%$ & $6^{\circ}$ \\
\hline BECR-Recog & $47.61(0.12)$ & $47.96(0.03)$ & $<0.002$ & $22.2 \%$ & $12^{\circ}$ \\
\hline \multicolumn{6}{|l|}{$\begin{array}{l}\text { Fluência verbal } \\
\text { COWAT }\end{array}$} \\
\hline \multicolumn{6}{|l|}{ Fonética } \\
\hline Letra "F" & $11.88(0.58)$ & $15.88(0.67)$ & $<0.001$ & $22.2 \%$ & $12^{\circ}$ \\
\hline Letra "A" & $10.83(0.48)$ & $14.14(0.63)$ & $<0.001$ & $25.9 \%$ & $11^{\circ}$ \\
\hline Letra "S" & $10.02(0.55)$ & $14.48(0.65)$ & $<0.001$ & $40.7 \%$ & \\
\hline Semântica & & & & & $1^{\circ}$ \\
\hline Animais & $16.35(0.56)$ & $19.61(0.73)$ & $<0.001$ & $18.5 \%$ & $18^{\circ}$ \\
\hline Frutas & $14.11(0.45)$ & $17.53(0.56)$ & $<0.001$ & $40.7 \%$ & 10 \\
\hline \multicolumn{6}{|c|}{ Pensamento abstrato } \\
\hline RAVEN & $16.89(0.55)$ & $18.70(0.58)$ & $<0.02$ & $27.8 \%$ & 8 \\
\hline
\end{tabular}

* Resultados dos testes neuropsicológicos nos pacientes com esclerose múltipla surto-remissão (EMSR) e no grupo controle com a percentagem de pacientes com resultados abaixo de $5 \%$ percentil e a ordem de maior comprometimento. A descrição dos testes se encontra no texto.

mento executivo ${ }^{10}$. A fluência verbal mostrou-se um teste altamente sensível para comprovar o comprometimento cognitivo na EM. Os presentes resultados, obtidos por meio dos testes RAVEN e COWAT, são de fato uma importante característica entre os pacientes com EMSR e estão de acordo com o perfil cognitivo já relatado na literatura, os quais relatam a cognição na esclerose múltipla como sendo uma desordem tipo subcortical executiva frontal $\left.\right|^{8,14}$.

Os resultados também indicaram que memória de longo prazo encontra-se significantemente comprometida em todos os estágios (aprendizagem, evocação e reconhecimento) nos testes RAVLT e BECR. A extensão do comprometimento da 
memória de longo prazo foi entre 18,5\%-29,6\% nos pacientes. Déficits de evocação em todas essas medidas estavam razoavelmente altas, na extensão de 30\%-35\%. Interessantemente, pacientes com EMSR apresentaram 22\% e 35\% de déficit no reconhecimento nos testes RAVLT e BECR, respectivamente. Esses resultados não estão em acordo com a visão clássica que assume que memória de reconhecimento está preservada em pacientes com esclerose múltipla ${ }^{8,14}$. Uma maior investigação será necessária para confirmar esse achado.

O teste que avaliou a velocidade de processamento de informações, o Digit Symbol, mostrou que 22,2\% dos pacientes apresentaram comprometimento. Esse tipo de teste foi apontado na literatura como sendo um dos mais sensíveis para detectar disfunção cognitiva em esclerose múltipla e a principal função cognitiva afetada nos pacientes ${ }^{29}$. No nosso caso, embora a frequência de alteração de velocidade de processamento de informação tenha sido alta (22,2\%), foi inferior ao índice de alteração de memória encontrado.

$O$ teste de rastreamento de demência (MIC) mostrou comprometimento em 9,3\% dos pacientes com EMSR, e os resultados do Digit Span indicaram que $11,1 \%$ desses pacientes apresentaram comprometimento no span atencional. Esses resultados são um tanto incoerentes com os relatos da literatura, os quais indicavam que pacientes com esclerose múltipla tinham span atencional preservado e menor pontuação de demência ${ }^{10,14}$. É possível que se tenha que rever os critérios de pontuação em testes de rastreamento para demência na população brasileira, como já tem sido feito ${ }^{30}$, visto que variáveis educacionais e culturais influenciam esses critérios.

\section{CONCLUSÃO}

De forma geral, os resultados foram concordantes com a literatura sobre cognição da esclerose múltipla, visto que as funções que apresentaram maior comprometimento foram as funções executivas, memória de longo prazo e velocidade de processamento de informação. As divergências encontradas se referem ao comprometimento da memória de reconhecimento, ao span atencional e ao rastreamento de demência, tendo sido encontradas alterações não comumente observadas na literatura internacional. Existem poucos estudos de levantamento das alterações cognitivas na esclerose múltipla na literatura brasileira. Portanto, serão necessários mais estudos para que se conheça o perfil das alterações cognitivas da esclerose múltipla no Brasil.

\section{REFERÊNCIAS}

1. Kantarci $\mathrm{OH}$, Weinshenker BG. Natural history of multiple sclerosis. Neurol Clin. 2005;23:17-38.

2. Kurtzke JF. Rating neurologic impairment in multiple sclerosis: an Expanded Disability Status Scale (EDSS). Neurology. 1983;33:1444-52.

3. Matthews EB. Clinical aspects. In: Compston DAS, editor. McAlpine's multiple sclerosis. London: Churchill Livingstone; 1998. p. 43-251.
4. Kutzelnigg A, Lucchinetti CF, Stadelmann C, Brück W, Rauschka H, Bergmann M, et al. Cortical demyelination and diffuse white matter injury in MS. Brain. 2005;128:2705-12.

5. Gaudino EA, Chiaravalloti ND, Deluca J, Diamond BJ. A comparison of memory performance in relapsing-remitting, primary progressive and secondary progressive, multiple sclerosis. Neuropsychiatry, Neuropsychol Behav Neurol. 2001;14:32-44.

6. Zakzanis KK. Distinct neurocognitive profiles in multiple sclerosis subtypes. Arch Clin Neuropsychol. 2000;15:115-36.

7. Riccitelli G, Rocca MA, Pagani E, Rodegher ME, Rossi P, Falini A, et al. Cognitive impairment in multiple sclerosis is associated to different patterns of gray matter atrophy according to clinical phenotype. Hum Brain Mapp. 2011. doi:10.1002/hbm. 21125.

8. Rao SM, Leo GJ, Bernardin L, Unverzagt F. Cognitive dysfunction in multiple sclerosis I: frequency, patterns and prediction. Neurology. 1991;41:685-91.

9. Prakash RS, Snook EM, Lewis JM, Motl RW, Kramer AF. Cognitive impairments in relapsing-remitting multiple sclerosis: a meta-analysis. Mult Scler. 2008;14(9):1250-61.

10. Bobholz JA, Rao SM. Cognitive dysfunction in multiple sclerosis: a review of recent developments. Cur Op Neurol. 2003;16:283-8.

11. Smestad C, Sandvik L, Landrø NI, Celius EG. Cognitive impairment after three decades of multiple sclerosis. Eur J Neurol. 2010;17(3):499-505.

12. Folstein MF, Folstein S, McHugh PR. 'Mini-Mental State'. A practical method for grading the cognitive state of patients for the clinician. J Psychol Res.1975;12:189-98.

13. Negreiros MAGM, Colin DD, Mattos PM, Papais-Alvarenga RM. FS Mental no EDSS: dificuldades no estadiamento cognitivo da esclerose múltipla. Arq Neuropsiq. 1996;54(Suppl):531.

14. Barget B, Camplais P, Borudette D. Cognitive dysfunction in multiple sclerosis: natural history, pathophysiology and management. CNS Drugs. 2002;16:445-55.

15. Benedict RH, Fischer JS, Archibald CJ, Arnett PA, Beatty WW, Bobholz J, et al. Minimal neuropsychological assessment of MS patients: a consensus approach. Clin Neuropsychol. 2002:16:381-97.

16. Beatty WW, Paul RH, Wilbanks SL, Hames KA, Blanco CR, Goodkin DE. Identifying multiple sclerosis patients with mild or global cognitive impairment using the Screening Examination for Cognitive Impairment (SEFCI). Neurology. 1995;45:718-23.

17. Ardila A. Directions of research in cross-cultural neuropsychology. J Clin Exp Neuropsychol. 1995; 17:143-50.

18. Andrade VM, Bueno OFA, Oliveira ASB, Oliveira EML, Miranda MC. Cognitive profile of patients with relapsing remitting multiple sclerosis. Arq Neuropsiq. 1999;57:775-83.

19. Paes RA, Alvarenga RM, Vasconcelos CC, Negreiros MA, Landeira-Fernández J. Neuropsicología de la esclerosis múltiple primaria progresiva. Rev Neurol. 2009;49(7):343-8.

20. Negreiros MA, Mattos P, Landeira-Fernandez J, Paes RA, Alvarenga RP. A brief neuropsychological screening test battery for cognitive dysfunction in Brazilian multiple sclerosis patients. Brain Inj. 2008;22(5):419-26.

21. Poser CM, Paty SW, Scheinberg L, McDonald WI, Davis FA, Ebers GC, et al. New diagnostic criteria for multiple sclerosis: guidelines for research protocols. Ann Neurol. 1983;13:227-31.

22. Blessed G, Tomlinson BE, Roth M. The association between quantitative measures of dementia and of senile change in the cerebral grey matter of elderly subjects. Br J Psych. 1968;114:797-811.

23. Negreiros MAGM, Papais-Alvarenga RM, Mattos PE. Alterações neurocognitivas na esclerose múltipla: resultados de primeiro estudo piloto. Arq Neuropsiq. 1996;54(Suppl):97.

24. Wechsler D. WAIS-III - Escala de Inteligência Wechsler para adultos. Adaptação e padronização de uma amostra brasileira. Elizabeth do Nascimento. São Paulo: Casa do Psicólogo; 2004.

25. Rey A. L'examen clinique em psychologie. Paris: Press Universitaires de France; 1964.

26. Buschke H. Cued recall in amnesia. J Clin Neuropsychol. 1984;6:433-40.

27. Benton AL, Hamsher K. Multilingual aphasia examination. lowa City: University of lowa Press; 1976.

28. Raven JC. Matrizes progressivas. Rio de Janeiro: CEPA - Centro Editor de Psicologia Aplicada; 1998.

29. Demaree HA, DeLuca J, Gaudino EA, Diamond BJ. Speed of information processing as a key deficit in multiple sclerosis: implications for rehabilitation. J Neurol Neurosurg Psych. 1999;67:661-3.

30. Brucki MD, Nitrini R, Caramelli P, Bertolucci PHF, Okamoto IH. Sugestões para 0 uso do Miniexame do Estado Mental no Brasil. Arq Neuropsiquiatr. 2003;61(3-B):777-81 